\title{
Frequência do vômito após cirurgia abdominal de urgência em hospital público de referência
}

\section{Frequency of vomiting after emergency abdominal surgery in a reference public hospital}

Fabiano Timbó Barbosa"; Roberta Ribeiro Marques Brandão²; Roberta louise Künzler A. de Almeida ${ }^{3}$

\section{R E S U M O}

\begin{abstract}
Objetivo: Avaliar a frequencia do vômito após cirurgia abdominal de urgência em hospital público de referência no Estado de Alagoas. Métodos: Após aprovação do Comitê de Ética em Pesquisa da Universidade Federal de Alagoas, os dados foram colhidos no prontuário médico 24 horas após a cirurgia abdominal de urgência. O teste de Fisher e o qui-quadrado com correção de Yates foram usados para comparar proporções. O intervalo de 95\% de confiança foi calculado quando possível. Resultados: Foram 100 pacientes, $85 \%$ homens (85/100, IC 95\% 0,78-0,92). A frequencia do vômito foi $25 \%$ sem diferença estatística entre cirurgia abdominal após trauma e abdome agudo inflamatório $(P=0,46)$. Não houve diferença estatística entre as técnicas anestésicas $(P=0,99)$. Conclusão: A frequencia do vômito no pós-operatório de cirurgia abdominal de urgência em hospital de referência no estado de Alagoas foi de $25 \%$
\end{abstract}

Descritores: Abdome/cirurgia. Vômito. Náusea e vômito pós-operatório. Complicações pós-operatórias.

\section{INTRODUÇÃO}

$A_{\text {sos- }}$ duas intercorrências mais frequentes observadas no pós-operatório de cirurgia abdominal são a náusea e o vômito ${ }^{1-6}$. Apesar dos avanços das técnicas anestésicas, do surgimento de fármacos de curta duração de ação e do desenvolvimento de novos antieméticos, a ocorrência global atualmente aceita situa-se entre $20-30 \%{ }^{4,7-9}$. Em populações de risco, essa incidência pode atingir $70 \%$ 6,8-10.

A ocorrência de náuseas e vômitos no pós-operatório (NVPO) traz diversas consequências de ordem médica e econômica, contribuindo para uma recuperação mais prolongada e consequentemente uma maior permanência no leito hospitalar ${ }^{1,4-6}$ exigindo internações não esperadas, um menor grau de satisfação do paciente e aumento dos custos hospitalares ${ }^{6,8-10}$. Aliados a esses fatores, existem as potenciais consequências orgânicas, como deiscência de suturas, aspiração pulmonar, pneumonia aspirativa, desidratação, alterações hidroeletrolíticas, rotura esofágica e aumento da pressão intracraniana ${ }^{1,4,6-8,10}$

O objetivo deste estudo foi avaliar a frequencia do vômito após cirurgia abdominal de urgência em hospital público de referência no Estado de Alagoas.

\section{MÉTODOS}

Tratou-se de um estudo observacional do tipo transversal realizado na Unidade de Emergência Dr. Armando Lages na cidade de Maceió. O estudo iniciou-se após a aprovação pelo Comitê de Ética e Pesquisa da Universidade Federal de Alagoas, através do processo $n^{\circ}$ 006138/2007-48, e da assinatura do termo de consentimento livre e esclarecido. Os critérios de inclusão foram: pacientes com escore de risco anestésico classificados como I, II e III da American Society of Anesthesiology de ambos os sexos, idade entre 18 e 60 anos, submetidos à cirurgia abdominal de urgência por trauma ou por abdome agudo e que receberam a anestesia geral.

O trabalho foi realizado no período de junho a outubro de 2007 sendo a amostra selecionada por covenciência. Os pacientes foram abordados 24 horas após o procedimento cirúrgico sobre a presença de náusea e vômito, assim como foi preenchido um formulário para extração dos dados do prontuário. A variável primária foi o vômito. As variáveis secundárias foram: idade, sexo, tipo de cirurgia, tipo de anestesia, medicações utilizadas e a náusea.

Utilizou-se a mediana assim como o valor máximo e mínimo para descrever a variável idade. Para a aná-

Trabalho realizado na Unidade de Emergência Doutor Armando Lages - Maceió- AL-BR.

1. Título Superior de Anestesiologia conferido pela Sociedade Brasileira de Anestesiologia e Mestre em Ciências da Saúde pela Universidade Federal de Alagoas - Maceió- AL-BR; 2. Médica Residente de Anestesiologia em terceiro ano do Centro de Ensino e Treinamento das Obras Sociais Irmã Dulce - Salvador -BA-BR; 3. Médica Residente de Radiologia e Diagnóstico por Imagenologia em terceiro ano do Conjunto Hospitalar do Mandaqui - São Paulo -SP-BR. 
lise das proporções foram utilizados o qui-quadrado com correção de Yates $\left(\mathrm{X}^{2}\right)$ e o teste exato de Fisher quando houve menos de 30 pacientes sob análise. O nível de significância adotado foi de 5\%. Foi calculado o intervalo de confiança de 95\% (IC 95\%) para as proporções. Foi utilizado o programa EPI Info para análise dos dados.

\section{RESULTADOS}

Participaram do estudo 100 pacientes, sendo $85 \%$ homens (85/100, IC 95\% 78 a 92\%) e 15\% mulheres (15/100, IC 95\% 0,11-0,19). A mediana da idade foi 32 anos, sendo a idade mínima 17 anos e a máxima 54 anos.

A frequencia de vômitos em todos os pacientes foi de $25 \%$ (25/100, IC 95\% 16 a 34\%). Dos pacientes do sexo masculino 21,2\% (18/85, IC 95\% 13 a 30\%) vomitaram, enquanto que entre as mulheres esta frequência foi de 46,7\% (7/15, IC 95\% 22 a 72\%), porém não houve diferença estatística significante entre os sexos $(P=0,075)$.

Em relação aos diagnósticos encontrados 64\% (64/100) foram vítimas de trauma abdominal aberto, 4\% (4/100) de trauma fechado e de 32\% (32/100) de abdome agudo inflamatório. A frequencia de vômitos dentre os pacientes com trauma abdominal e submetidos à laparotomia foi de 27,9\% (19/68 IC 95\% 17 a 39\%). A frequencia de vômitos em vítimas de abdome agudo inflamatório foi de 18,7\% (6/32 IC 95\% 5 a 32\%), porém não houve diferença estatística significante entre os diferentes diagnósticos $(\mathrm{P}=0,46)$.

Considerando o tipo de anestesia, a anestesia geral balanceada com óxido nitroso foi utilizada em $80 \%$ (80/100), a anestesia geral balanceada sem óxido nitroso em 15\% (15/100) e anestesia geral venosa total em 5\% (5/100). A frequencia de vômito nos pacientes submetidos à anestesia geral balanceada com e sem óxido nitroso foi de 25,3\% (24/95 IC 95\% 17 a 34\%) e da anestesia geral venosa foi de $20 \%$ (1/5 IC 95\% - 15 a 55\%), porém não houve diferença estatística significante entre os vários tipos de anestesia geral $(P=0,99)$.

Considerando os pacientes que se submeteram a anestesia geral balanceada, 26,3\% (21/80 IC 95\% 17 a $33 \%$ ) vomitaram com o uso de óxido nitroso e $20 \%$ (3/15 IC $95 \%$ a $40 \%$ ) sem o uso de óxido nitroso não havendo diferença estatística significante entre os tipos de anestesia balanceada $(\mathrm{P}=0,44)$.

Com relação às drogas utilizadas no intra-operatório em 69\% (69/100) dos pacientes não foi utilizada nenhuma droga, $21 \%$ (21/100) metoclopramida, 4\% (4/100) dexametasona, 3\% (3/100) bromoprida e em 1\% (1/100) houve a combinação de metoclopramida e dexametasona.

Em relação ao pós-operatório, foram utilizadas drogas antieméticas em 85\% (85/100) dos pacientes e nenhuma droga em 15\% (15/100). A metoclopramida foi utilizada em 92,94\% (79/85), a bromoprida em 5,88\% (5/
85) e associação ondansetron com metoclopramida em $1,17 \%$ (1/85). A frequencia de vômitos entre os que receberam de metoclopramida foi de 26,6\% (21/79 IC 95\% 17 a 36\%) não havendo diferença estatística significante $(P=0,71)$ entre os pacientes que utilizaram metoclopramida e os pacientes que usaram bromoprida. A frequencia de vômitos nos pacientes que não usaram nenhuma droga anti-emética no pós-operatório foi de 26,7\% (4/15 IC 95\% 4 a 49\%) que em comparação com a metoclopramida 26,6\% (21/79 IC 95\% 17 a 36\%) não houve diferença estatística significante $(P=0,60)$.

A frequencia de náuseas no pós-operatório foi de $41 \%$ (41/100 IC 95\% 31 a 51\%). Destes, 80,5\% (33/ 41) estavam sob uso de metoclopramida, 4,9\% (2/41) receberam bromoprida e 14,6\% (4/41) não estavam recebendo medicação para náusea. Não houve diferença estatística significante $(P=0,87)$ entre os pacientes que não receberam medicação para náusea e aqueles que receberam metoclopramida.

A maior freqüência de vômitos ocorreu em pacientes vítimas de trauma abdominal aberto $(29,7 \%)$ em relação aos pacientes de abdome agudo inflamatório $(18,7 \%)$, porém não houve diferença estatística significante entre eles $(P=0,46)$.

\section{DISCUSSÃO}

A etiologia do vômito é considerada multifatorial e depende das características do paciente, do tipo e da duração da anestesia e do procedimento cirúrgico realizado $^{1,2,4,6,8-12}$. Os fatores de risco já bem estabelecido e que se correlacionam com o paciente são: sexo, idade, história prévia de NVPO, cinetose e antecedentes de tabagismo ${ }^{5,6,9-}$ 12. Os fatores relacionados à cirurgia são: local e a duração da cirurgia e os fatores relacionados à anestesia são: ventilação sob máscara e utilização de drogas com potencial emetogênico ${ }^{11}$. Há ainda fatores relativos ao pós-operatório, como: dor, o transporte do paciente à sala de recuperação e a deambulação precoces que aumentam esse ris$\mathrm{CO}^{1,4}$

Vários estudos têm sido efetuados com o intuito de melhor esclarecer os fatores de risco e assim evitá-los ou tratá-los, mas o modelo preditivo mais utilizado é o de APFEL ${ }^{13}$ que baseia-se em cinco fatores de risco: sexo feminino, história de náuseas e vômitos ao movimento, história de NVPO, ausência de tabagismo e uso pós-operatório de opióides ${ }^{6,8,10}$. A ocorrência de NVPO seria de 10\%, $21 \%, 39 \%, 61 \%$ e $79 \%$ respectivamente se $0,1,2,3$ ou 4 destes fatores de risco estiverem presentes ${ }^{3,6,9,10}$

O uso de sonda nasogástrica após cirurgias abdominais foi uma rotina durante muitos anos pela impressão de que a descompressão gástrica levaria a uma menor taxa de complicações. No entanto, esta teoria vem sendo desmistificada após vários estudos mostrando que a retirada precoce ou mesmo a ausência de sonda nasogástrica 
após operações abdominais não está associada a uma maior ocorrência de complicações ${ }^{14}$.

Em nosso estudo o número de homens foi superior ao número de mulheres, $85 \%$, e isso poderia ter comprometido os resultados uma vez que o vômito é mais frequente no sexo feminino, entretanto este elevado número de homens pode ser justificado pela maior frequência estatística de homens envolvidos em traumas na população em geral na proporção de 2,5:115. A análise estatística evidenciou não haver diferença entre os sexos nesta pesquisa $(0,075)$

A média das idades dos pacientes deste estudo foi de 29,18 anos e aproximadamente $68 \%$ dos pacientes apresentaram idade entre 19,1 anos e 39,3 anos. Esta faixa etária não se apresenta por si só como um fator de risco, uma vez que a incidência de NVPO nos adultos está em torno de $25-30 \%$, enquanto em crianças é cerca de $40 \% 1,6,8,9,10$, podendo chegar a $51 \%$ nos pacientes entre 6 e 16 anos $^{9}$.

Todos os pacientes do estudo foram submetidos à laparotomia devido a trauma abdominal (aberto ou fechado) ou abdome agudo inflamatório. A literatura relata maior incidência de NVPO após operações abdominais, laparoscopia, cirurgias ortopédica, ginecológica, otorrinolaringológica, cirurgias de mama, cirurgias plásticas, bem como neurocirurgias ${ }^{1,3,4,8,9}$. Entretanto ainda não existe consenso em relação aos dados de pacientes envolvidos em trauma abdominal e abdome agudo inflamatório.

Numerosas variáveis anestésicas têm sido bem estabelecidas como fatores de risco para NVPO incluindo o uso de anestésicos voláteis, do óxido nitroso, da anestesia geral balanceada e de largas doses de neostigmina ${ }^{8}$. Neste estudo foi comparado o uso de anestesia geral balanceada com e sem óxido nitroso, com a venosa total observando as taxas de NVPO. Aquela que levou a uma maior ocorrência de NVPO foi à anestesia balanceada com óxido nitroso, porém não houve diferença estatística significante quando comparada com a anestesia venosa total $(P=0,99)$. O mecanismo exato que justifique o óxido nitroso como indutor do vômito não é completamente esclarecido ${ }^{15}$. As principais proposições incluem um me- canismo central e um periférico através da sua ação em espaços fechados como a orelha média e o trato gastrintestinal ${ }^{15}$.

Alguns estudos têm enfatizado que as intervenções para prevenir NVPO não são recomendadas para pacientes sem nenhum fator de risco ${ }^{8}$ ou para pacientes classificados como baixo risco ${ }^{9}$. A identificação de pacientes de alto risco para NVPO permite uma profilaxia mais eficaz considerando combinações de duas ou três drogas protetoras $^{8}$. Nos pacientes de moderado risco pode ser necessária uma medicação ou combinação de duas drogas $^{8,9}$. Neste estudo, apenas $31 \%$ dos pacientes recebeu medicação no intra-operatório e 69\% permaneceu sem essa profilaxia.

Nesse estudo 85\% dos pacientes receberam droga antiemética no pós-operatório sendo a mais utilizada a metoclopramida. A ocorrência de vômitos no pós-operatório foi de $26,6 \%$. Dentre os pacientes que não receberam a profilaxia antiemética, 15\%, houve vômitos em 26,7\% não havendo diferença estatística significante em comparação com aqueles que receberam a metoclopramida $(P=0,60)$. Segundo a literatura, $50 \%$ dos estudos mostram que a metoclopramida não é mais eficiente que o placebo ${ }^{16}$ e quando usada nas doses clínicas (10mg endovenosa), ou até mesmo em altas doses, não se mostrou mais eficaz ${ }^{10}$. Segundo o consenso de manejo de náusea e vômitos no pós-operatório publicado em $2003^{10}$, se náuseas e vômitos ocorrerem até seis horas de pós-operatório, os pacientes não devem receber uma dose repetida do mesmo medicamento usado na profilaxia. Nessa ocasião, uma droga de outra classe deverá ser usada ${ }^{10}$. Após seis horas de pósoperatório, os pacientes podem ser tratados com qualquer agente usado na profilaxia, exceto a dexametasona e a escopolamina transdérmica ${ }^{10}$. No nosso estudo, após a ocorrência de vômitos, $72 \%$ dos pacientes continuaram sob o uso da metoclopramida, 4\% com bromoprida, e em $24 \%$ não foi utilizada nenhuma droga para o tratamento apesar do vômito já instalado.

Os resultados obtidos neste estudo permitem concluir que a frequencia do vômito em pós-operatório de cirurgia abdominal de urgência em hospital de referência no estado de Alagoas foi de $25 \%$.

\section{ABSTRACT}

Objective: The objective of this study was to evaluate the frequency of the vomiting after urgency abdominal surgery in the Alagoas reference public hospital. Methods: After approved in the Ethic and Search Committee of the Alagoas Federal University, the information was got in the medical records 24 hours after urgency abdominal surgery. Fisher test and chi-square with Yates correction were used do compare proportions assuming a 5\% significance level. Was calculated $95 \%$ confidance interval to proportions when was possible. Results: Were 100 patients, 85\% men (85/100, Cl 95\% 0,78-0,92). The vomiting frequency was $25 \%$ without statistical difference between abdominal surgery after trauma and inflammatory acute abdome (P=0,46). There was not statistical difference between anesthetic techniques $(P=0,99)$. Conclusion: The general vomiting prevalence after the urgency abdominal surgery in the Alagoas reference public hospital was $25 \%$.

Key words: Abdomen/surgery. Vomiting. Postoperative nausea and vomiting. Postoperative complications. 


\section{REFERENCIAS}

1. Carvalho WA, Viana PTG, Braz JRC. Náuseas e vômitos em anestesia: Fisiopatologia e Tratamento. Rev Bras Anestesiol. 1999; 49(1):65-79.

2. Martins RS, Martins ALC, Grillo FDL, Bartolozzo CR. Prevenção de Náuseas e Vômitos no pós-operatório com Ondasetron: comparação com prometazina. Rev Bras Anestesiol. 1995; 45(4):253-8.

3. Bedin A, Pinho MSL, Zanotelli CT, Caldart AS, Turazzi JC, Castro RAC. Dexametasona comparada à metoclopramida na profilaxia de vômitos pós-operatórios em crianças submetidas a procedimentos cirúrgicos ambulatoriais. Rev Bras Anestesiol. 2005; 55(4):387-96

4. Schimidt A, Bagatini A. Náusea e vômito pós-operatório: fisiopatologia, profilaxia e tratamento. Rev Bras Anestesiol. 1997; 47(4):326-34

5. Fonseca NM, Cardoso RCGP. Prevenção de náuseas e vômitos com metoclopramida em pacientes submetidos a curetagem uterina sob anestesia geral. Rev Bras Anestesiol. 2001; 51(2):10511.

6. Lages N, Fonseca C, Neves A, Landeiro N, Abelha FJ. Náuseas e vômitos no pós-operatório: uma revisão do "pequeno - grande" problema. Rev Bras Anestesiol. 2005; 55(5):575-85.

7. Lewaschiw EM, Pereira IA, Amaral JLG. Ondansetrona oral na prevenção de náuseas e vômitos pós-operatórios. Rev Assoc Med Bras 2005; 51(1):35-40.

8. Gan TJ. Risk factors for postoperative nausea and vomiting. Anesth Analg 2006; 102(6):1884-98

9. Habib AS, Gan TJ. Evidence-based management of postoperative nausea and vomiting: a review. Can J Anesthes. 2004; 51(4):32641.

10. Gan TJ, Meyer T, Apfel CC, Chungs F, Davis PJ, Eubanks S et al. Consensus guidelines for managing postoperative nausea and vomiting. Anesth Analg. 2003; 97(1):62-71.

11. Abreu MP, Vieira JL, Silva IF, Miziara LE, Fófana R. Eficácia do ondasetron, metoclopramida, droperidol e dexametasona na pre- venção de náusea e vômito após laparoscopia ginecológica em regime ambulatorial. Rev Bras Anestesiol. 2006; 56(1):8-15.

12. Ganem EM, Fabris P, Moro MZ, Castiglia YMM. Eficácia do ondansetron e da alizaprida na prevenção de náusea e vômito em laparoscopia ginecológica. Rev Bras Anestesiol. 2001; 51(5):401-6.

13. Apfel CC, Korttila K, Abdalla $M$ et al - A factorial trial of six interventions for the prevention of postoperative nausea and vomiting. N Engl J Med, 2004;350:2441-51.

14. Andrade AC, Barjud MB, Cavalcante JM, Matos LAN. Anastomose bílio-digestiva sem descompressão gástrica. Rev Col Bras Cir. 2006; 33(3):242-4

15. Crawford MW, Lerman J, Sloan MH, Sikich N, Halpern L, Bissonnette B. Recovery characteristics of propofol anaesthesia, with and without nitrous oxide: a comparison with halothane/nitrous oxide: a comparison with halothane/nitrous oxide anaesthesia in children. Paediatr Anaesth. 1998; 8(1):49-54.

16. Polati E, Verlato G, Finco G, Mosaner W, Grosso S, Gottin L et al. Ondansetron versus metoclopramide in the treatment of postoperative nausea and vomiting. Anesth Analg. 1997; 85(2):3959.

Recebido em 24/04/2009

Aceito para publicação em 01/07/2009

Conflito de interesse: nenhum

Fonte de financiamento: nenhuma

\section{Como citar esse artigo:}

Barbosa FT, Brandão RRM, Almeida RLK. Frequência do vômito após cirurgia abdominal de urgência em hospital público de referência. Rev Col Bras Cir. [periódico na Internet] 2010; 37(4). Disponível em URL: http://www.scielo.br/rcbc

\section{Endereço para correspondência:}

Fabiano Timbó Barbosa

E-mail: fabianotimbo@yahoo.com.br 